University of Wollongong

Research Online

Faculty of Engineering and Information

Faculty of Engineering and Information

Sciences - Papers: Part A

Sciences

$1-1-2006$

Stereoscopic visualization and editing of automatic abdominal aortic aneurysms (AAA) measurements for stent graft planning

Luping Zhou

Volume Interactions Pte Ltd, lupingz@uow.edu.au

Yaping Wang

Volume Interactions Pte Ltd

Lin-Chia Goh

Volume Interactions Pte Ltd

Ralf A. Kockro

Volume Interactions Pte Ltd

Luis Serra

Volume Interactions Pte Ltd

Follow this and additional works at: https://ro.uow.edu.au/eispapers

Part of the Engineering Commons, and the Science and Technology Studies Commons

Research Online is the open access institutional repository for the University of Wollongong. For further information contact the UOW Library: research-pubs@uow.edu.au 


\title{
Stereoscopic visualization and editing of automatic abdominal aortic aneurysms (AAA) measurements for stent graft planning
}

\author{
Abstract \\ For stent graft selection in the treatment of abdominal aortic aneurysms (AAA) anatomic considerations \\ are important. They determine GO/NO-GO of the treatment and help customize the stent. Current systems \\ for AAA stent insertion planning based on pre-operative CT and MR of the patient do not provide an \\ intuitive interface to view the resulting measurements against the pre-operative CT/MR. Subsequent \\ modifications of the measurements are frequent when automatic algorithms are inaccurate. However, 3D \\ editing is difficult to achieve because of the limitations of monoscopic displays and 2D interface. In this \\ paper, we present a system for automatic AAA measurement and interactive 3D editing. The strength of \\ this approach is that the resulting measurements can be reviewed and edited interactively in the 3D \\ context of the volumetric rendering of the aorta, so that relationships of the measurements and the aorta \\ are clearly perceived. This understanding is facilitated by the stereoscopic rendering that makes it \\ possible to see the transparent vessel and its corresponding measurements all in one image.
}

\section{Keywords}

graft, planning, abdominal, automatic, aortic, editing, visualization, stereoscopic, aneurysms, aaa, measurements, stent

\section{Disciplines \\ Engineering | Science and Technology Studies}

\section{Publication Details}

Zhou, L., Wang, Y., Goh, L., Kockro, R. A. \& Serra, L. (2006). Stereoscopic visualization and editing of automatic abdominal aortic aneurysms (AAA) measurements for stent graft planning. In A. J. Woods, N. A. Dodgson, J. O. Merritt, M. T. Bolas \& I. E. Mcdowall (Eds.), Proceedings of SPIE - Stereoscopic Displays and Virtual Reality Systems XIII (pp. 605507-1-605507-9). San Jose, United States: SPIE. 


\title{
Stereoscopic Visualization and Editing of Automatic Abdominal Aortic Aneurysms (AAA) Measurements for Stent Graft Planning
}

\author{
Luping Zhou, Yapeng Wang, Lin-Chia Goh, Ralf A Kockro and Luis Serra \\ Volume Interactions Pte. Ltd., Singapore, 237994
}

\begin{abstract}
For stent graft selection in the treatment of abdominal aortic aneurysms (AAA) anatomic considerations are important. They determine GO/NO-GO of the treatment and help customize the stent. Current systems for AAA stent insertion planning based on pre-operative $\mathrm{CT}$ and $\mathrm{MR}$ of the patient do not provide an intuitive interface to view the resulting measurements against the pre-operative CT/MR. Subsequent modifications of the measurements are frequent when automatic algorithms are inaccurate. However, 3D editing is difficult to achieve because of the limitations of monoscopic displays and 2D interface. In this paper, we present a system for automatic AAA measurement and interactive 3D editing. The strength of this approach is that the resulting measurements can be reviewed and edited interactively in the $3 \mathrm{D}$ context of the volumetric rendering of the aorta, so that relationships of the measurements and the aorta are clearly perceived. This understanding is facilitated by the stereoscopic rendering that makes it possible to see the transparent vessel and its corresponding measurements all in one image.
\end{abstract}

Keywords: Abdominal Aortic Aneurysms, Stent Graft Planning, Automatic Measurements, Interactive Stereo 3D Editing, Volume Rendering, Dextroscope ${ }^{\mathrm{TM}}$

\section{INTRODUCTION}

The treatment of abdominal aortic aneurysms (AAA) by means of the implantation of a stent through the femoral artery is a procedure that has reached wide clinical acceptance. Anatomical considerations are important in stent graft selection. They can determine the GO/NO-GO of the treatment and help customize the stent. For example, since the stent is inserted through the femoral artery, the access arteries (the external and common iliac) must be large enough to accept the stent and must exceed 7.0 millimeters in diameter.

Conventionally, AAA systems measure using the acquired 2D slices, the orientation of which is not necessarily orthogonal to the blood vessel. However, AAAs are usually both tortuous and eccentric. These properties can corrupt the measurement accuracy on conventional 2D CT images [1]. Hence, 3D imaging and analysis techniques are increasingly used in AAA systems. The prevalent method is to find the centerline of the blood vessel and to measure it, along with the diameter and area at certain key points of the vessel lumen in a plane perpendicular to the axis (the "true" cross-section). However, it is still difficult to edit these measurements and exhibit them in a way that is clear and unambiguous.

There exists several AAA stent insertion planning systems. They measure the optimal diameters and lengths based on pre-operative CT and MR and display the measurements in 2D interface [2][3][4]. Though most of these systems employ multiplanar reconstruction to display the aorta in axial, sagittal, coronal views simultaneously and some also provide a 3D view (monoscopic), but due to the lack of depth perception of the monoscopic image there is significant loss of the spatial information for the data and the measurements . To overcome the shortcoming of monoscopic display for 3D context, these systems try to reconstruct the whole 3D picture by combining pieces of 2D information. For example, Philips EasyVision Workstation measures the diameters and displays the measurements in two panels. One shows the construction of the central lumen line of the aorta in a sagittal plane.

Stereoscopic Displays and Virtual Reality Systems XIII, edited by Andrew J. Woods, Neil A. Dodgson, John O. Merritt, Mark T. Bolas, Ian E. McDowall, Proc. of SPIE-IS\&T Electronic Imaging, SPIE Vol. 6055, 605507, (c) 2006 SPIE-IS\&T $\cdot 0277-786 \times / 06 / \$ 15$ 
The other shows the cross-section of the aorta perpendicular to the central lumen line (indicated in the first panel) and its corresponding diameter measurement. Barco's Voxar 3D VesselMetrix works in another way. It simultaneously rotates the curved vessel and the $360^{\circ}$ straightened vessel view to provide characterization and navigation. However, these methods are not intuitive. Users have to "assemble" the information in their mind in order to obtain a complete understanding of the anatomy. The ideal method should allow users to view directly the resulting measurements against the pre-operative CT/MRI, so that they can check the measurement accuracy in 3D context. Moreover, modification of the measurements are frequent when automatic algorithms introduce inaccuracies (the diameter of the thrombus, for instance, is difficult to detect due to its low contrast). However, 3D editing is difficult to achieve too because of the limitations of monoscopic displays and the 2D interfaces in these systems.

In this paper, we present AngioDEX, a system for automatic AAA measurements and editing within a stereoscopic virtual environment. Facilitated by stereoscopic rendering, AngioDEX provides users a clear understanding of the spatial relationship between the measurements and the abdominal aorta aneurysm and an easy way to precisely edit the measurements in 3D.

\section{METHODOLOGY}

\subsection{Overview}

AngioDEX is constructed based on the Dextroscope ${ }^{\circledR}$ - a two-handed 3D stereoscopic virtual environment for working with medical images [5][6][7]. The Dextroscope introduces a mirror between the user and the computer screen, so that the resulting image, when perceived with stereoscopic glasses, appears to be floating within the user's reach. Hence, the virtual object appearing on the screen can be reached into with a 3D input device. Users can interact with the 3D volume precisely through 3D tracking devices on each hand; the right hand holds a penlike stylus, while the left holds a joystick-like 6D controller.

AngioDEX consists of two main modules; an automatic measurement of anatomical parameters and an interactive editing of the measurements. In automatic measurement, AngioDEX requires four input points, and produces both parametric measurements (diameter and length) and structural information (location of bifurcations). In 3D editing, AngioDEX allows users to move, rotate, resize and reshape the diameter measurements and ensures the consistency of the length measurements.

\subsection{Initial placement of the input points}

To start the automatic measurement, the user inputs four points inside the MR or CT of an aorta corresponding to the points above and below the stent anchoring points, and the two ends of iliac arteries, indicating the region of interest (Figure $1 \mathrm{~b}$ ). In traditional AAA planning products, users have to input the points in 2D slices. When the aorta is tortuous, sometimes it is difficult to tell the right position (for example, "just below the renal artery") in 3D only from 2D information. But in AngioDEX, with the 3D tracking device it is very easy to "reach" into the stereoscopic aorta and place the points. Yet there is still a small problem of this operation: the user's tool may go through outside the aorta without arousing the user's attention because the aorta blocks his view. To solve this problem, AngioDEX introduces a Slice Viewer which displays the cross-section of the aorta along the user's tool. By monitoring the Slice Viewer, the user is aware of the boundary of the aorta (Figure 1 a). 


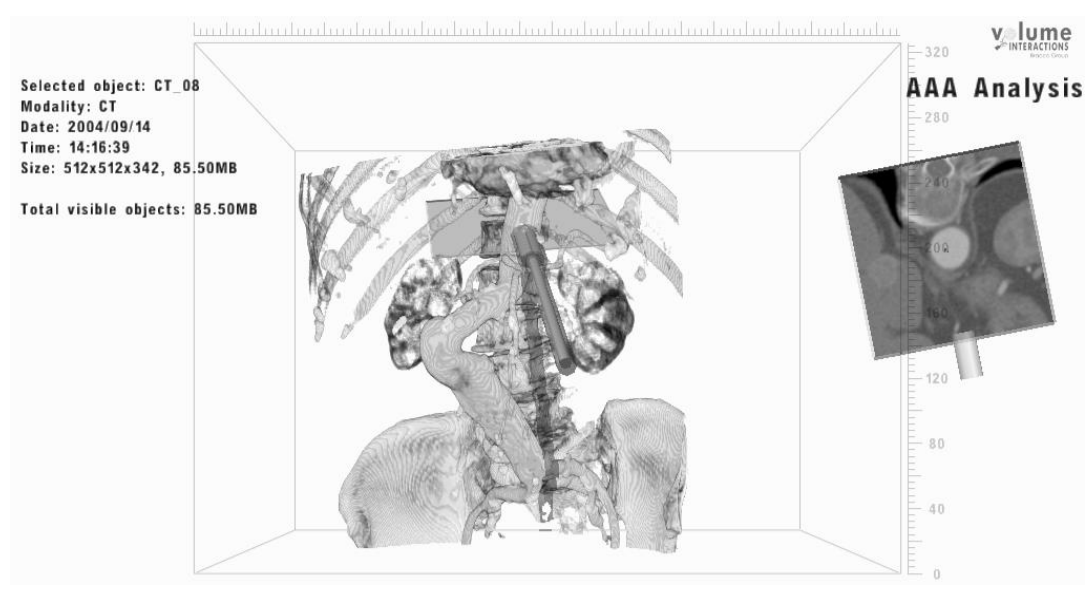

(a)

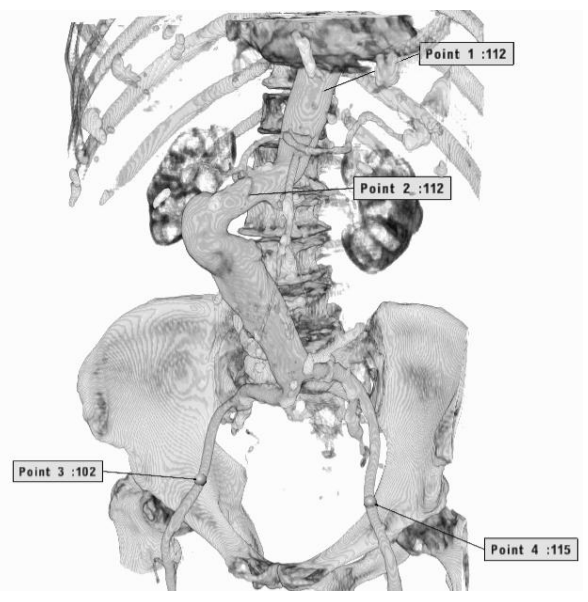

(b)

Figure 1: Placement of initial points for automatic AAA measurement.

(a) Guidance with Slice Viewer and (b) the four user-defined points defining the region of interest.

\subsection{Automatic measurement and display}

The automatic measurement consists of four steps (Figure 2): Aorta Segmentation, Lumen Centerline Extraction, Ellipse Mapping (diameter measurements) and Template Mapping (structural information and length measurements).

In the aorta segmentation, an adaptive region growing method is used to segment the aorta within the region of interest. The edge points detected by edge detection are used as constrains for the region growing from the seed points. In the lumen centerline extraction, the skeleton of the segmented aorta is extracted by a morphological thinning algorithm. Given the start and end points, the centerline is then generated by tracking between the points on the skeleton. During ellipse mapping, the segmented aorta is re-sampled to generate a cross-section orthogonal to the centerline. Ellipse mapping is applied throughout the whole length of the centerline to provide information for template mapping. The step of template mapping locates the key measurements required by a general AAA stent insertion planning process. A predefined structural template describes the general structure of the AAA that will be mapped onto the scanning data by extracting and matching features like anatomical bifurcations of aorta and iliac arteries.

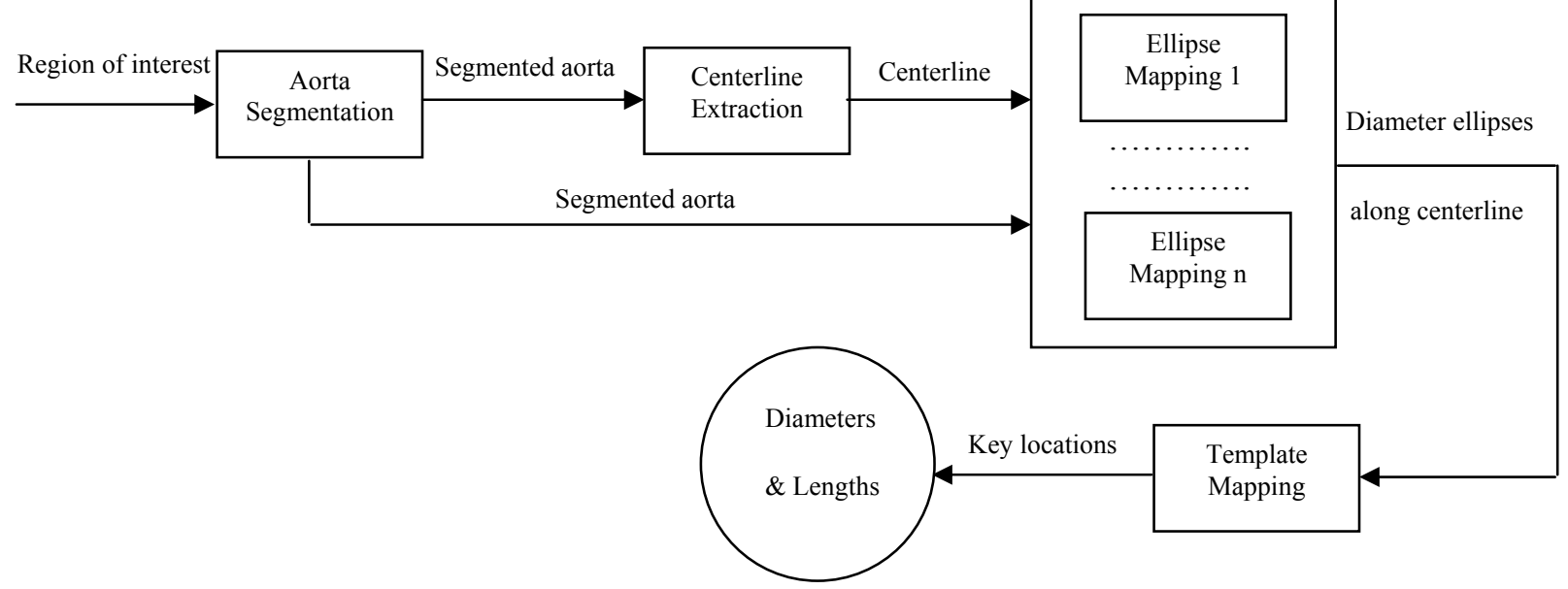

Figure 2: Workflow of automatic measurement. 
There are two main outcomes of the automatic measurement: the lumen centerline of the aorta within the region of interest and a structured template with measurements for this particular aorta (Figure 3 a). The centerline is represented as a piece-wise B-spline curve. It is divided into eight sections with different color codes according to the extracted structural information. The structured template contains measurements of diameters for most critical points along the aorta (bifurcations, minimum and maximum points) plus lengths between those points. A diameter measurement is represented as an ellipse orthogonal to the lumen centerline to approximate the perimeter of the cross-section. The long axis and short axis of the ellipse represent the maximum and minimum cross-sectional diameters respectively. A length measurement is computed along the curved centerline and discriminated by their color codes. Each measurement has an associated label to indicate its values.

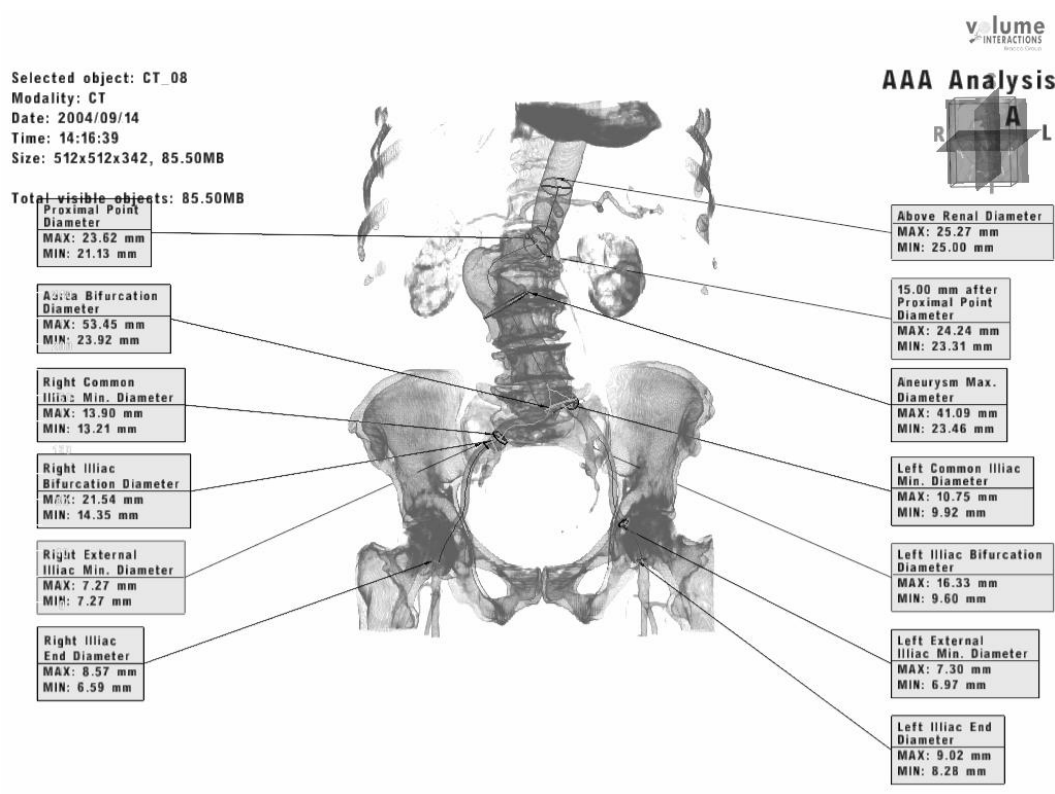

(a)

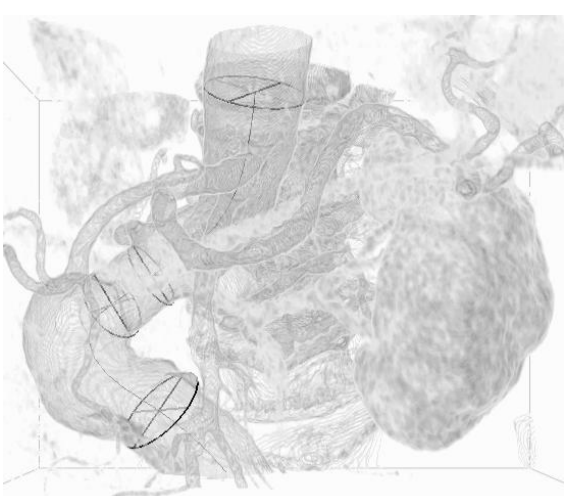

(b)

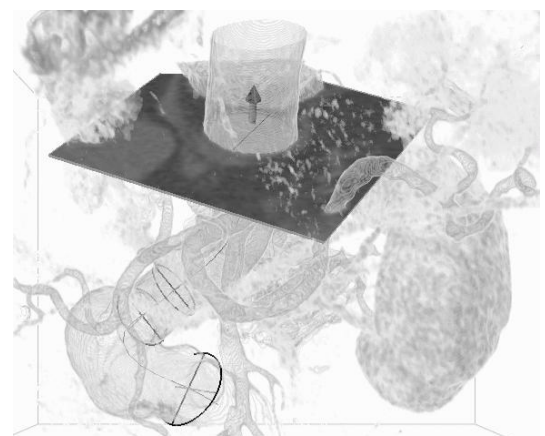

(c)

Figure 3: Results of automatic measurements. (a) The derived structural template, (b) a close observation of the diameter measurements against the aorta and (c) a close observation of the diameter measurements with the 2D reference image.

The strength of this approach is that the resulting measurements can be viewed against the volume rendering of the aorta, so that the relationship of the measurements and the aorta are clearly perceived (Figure $3 \mathrm{~b}$ ). Users can understand where these measurements are in their 3D context and how accurate these measurements are by checking the fit of the ellipse to the aneurysm volume. This understanding is facilitated by the stereoscopic rendering that makes it possible to see the transparent vessel and its corresponding measurements all in a single image. Alternatively, users can also check the fit of the ellipse by using the 2D reference image when they are editing the diameter measurements. A reference image is an interpolated slice intersecting with the volume of the aorta, showing the original intensities (Figure $3 \mathrm{c}$ ).

The stereoscopic visualization gives the system a lot of possibilities to visually validate the measurements. For example, users can "fly" through the inside of the vessel guided by the centerline and keep the vessel wall semitransparent, hence seeing the inside and outside of the vessel as well as the measurement. This is possible thanks to the combination of stereoscopy and degrees of transparency. 


\subsection{D editing of measurements}

AngioDEX provides a set of 3D editing tools to edit the measurements in a virtual environment. Users can adjust the centerline, move, rotate, resize and reshape the diameter measurements. After editing, AngioDEX ensures the structural consistency of the template.

\subsubsection{Adjusting the centerline}

AngioDEX can map the template correctly onto most tortuous aortas. However, in some difficult cases, the aortas can be very tortuous and possibly even form a loop as a result of an improper scan. In such cases, the user can choose to manually fine-tune the centerline by selecting the "Edit Centerline" tool. A few levels of user selectable controls are available to adjust the "magnetism" of the tool. This tool also allows the user to extend the input points that were placed prior to the automatic computation. The length measurements are computed once the adjustment of the centerline is finalized.

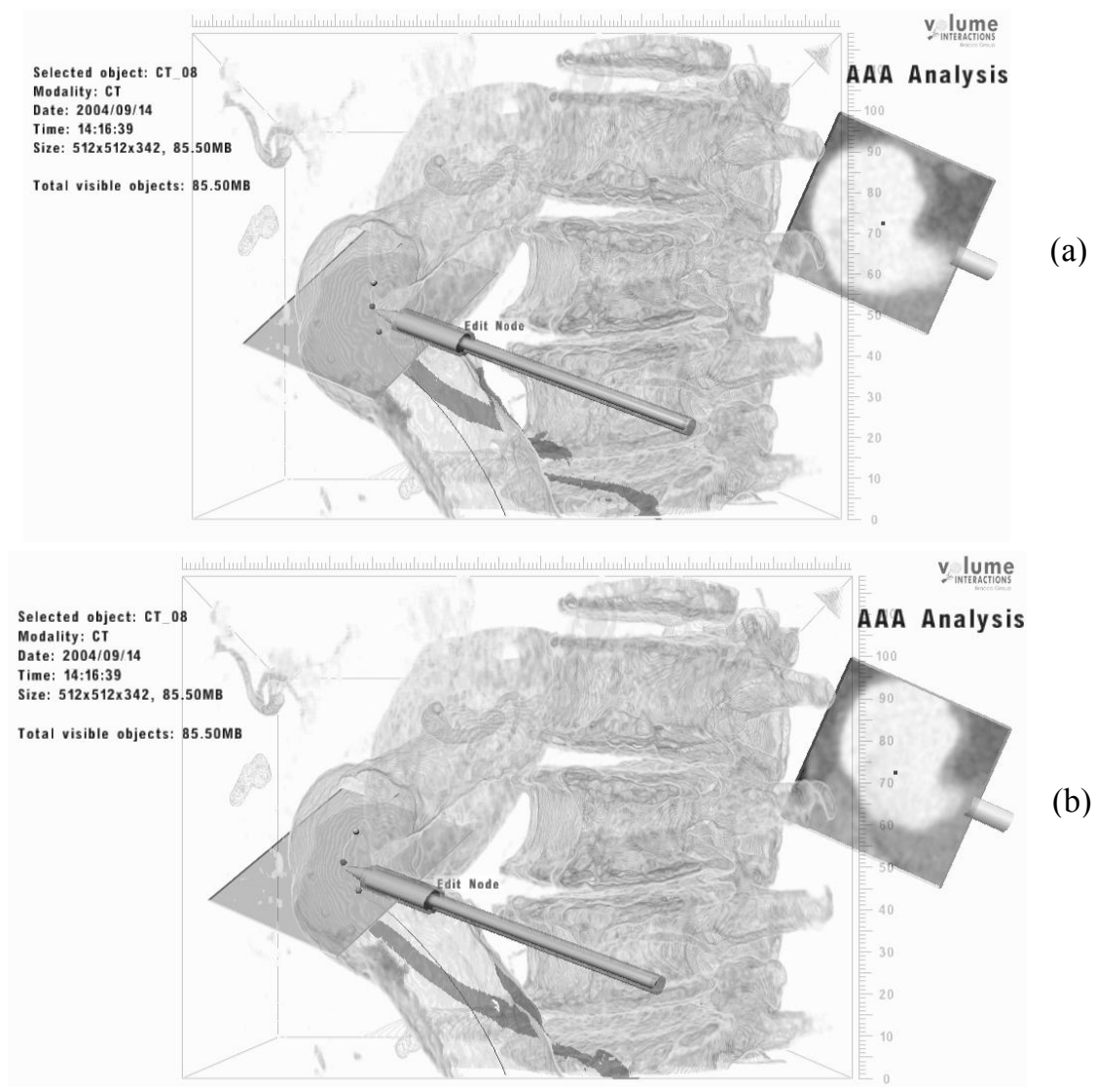

Figure 4: Editing the centerline. (a) Centerline before editing. A point in the centerline (critical point) is selected for editing. The surrounding points are affected by the movement of the critical point. The Slice Viewer is used to guide the editing. (b) Centerline after editing. The centerline is modified to have a better fit of the aorta.

\subsubsection{Moving the diameter measurements}

To move the diameter measurements in AngioDEX, the user selects the "Move" tool and simply moves the virtual tool near to the displayed measurement and performs a "click and drag" action. When the tool approaches any diameter measurements, a 2D reference image corresponding to the position of the diameter measurement is shown to indicate that that is the current active slice for editing. The user may move the measurement along the centerline 
and is only restricted in position by the two (superior and inferior) diameter measurements along the centerline. Once the user has determined the new position, AngioDEX automatically computes the new diameter. If the movement of the diameter involves a measurement that borders two length measurements, for example, at the aorta bifurcation, the length measurements would also be automatically updated.

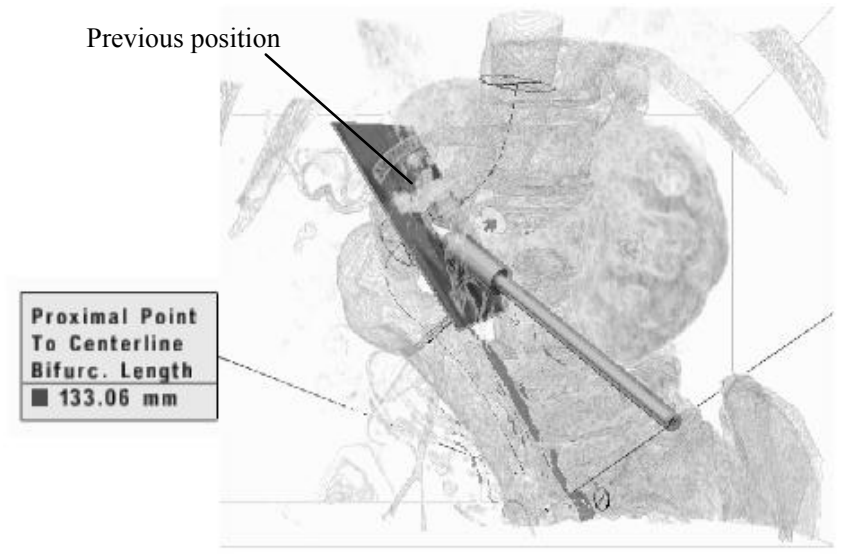

(a)

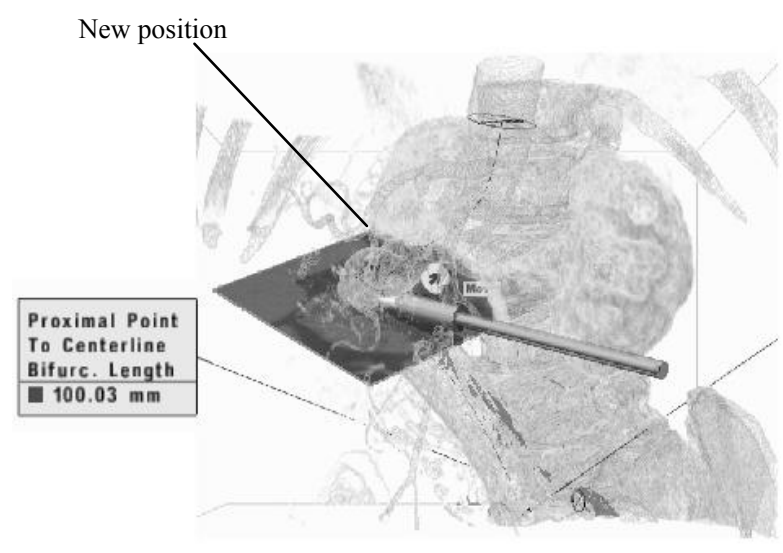

(b)

Figure 5: Moving the diameter ellipse. (a) Before moving, the green line indicates the ellipse to be moved. (b) After moving, the ellipse is moved further from the lower renal artery. The length of the red line is automatically updated from $133.06 \mathrm{~mm}$ to $100.03 \mathrm{~mm}$.

\subsubsection{Rotating the diameter measurements}

Sometimes, the best diameter measurements cannot be measured orthogonal to the centerline, especially, in cases where the aorta is very tortuous. To measure the diameter of the aorta at an oblique plane, the user switches to the "Rotate" tool. The 2D reference image acts as a placeholder for the user to freely rotate the diameter measurements about the position on the centerline (Figure 6). This ability of rotating the diameter measurements to any oblique orientation is something that cannot be easily achieved, especially, with 2D packages. The ease of control, coupled with the intuitive interface allows almost instantaneous editing of the orientation of the diameter measurements and is one of the biggest strengths of AngioDEX. Once the user determines the correct orientation of the measurement, AngioDEX recomputes the new diameters at that orientation.

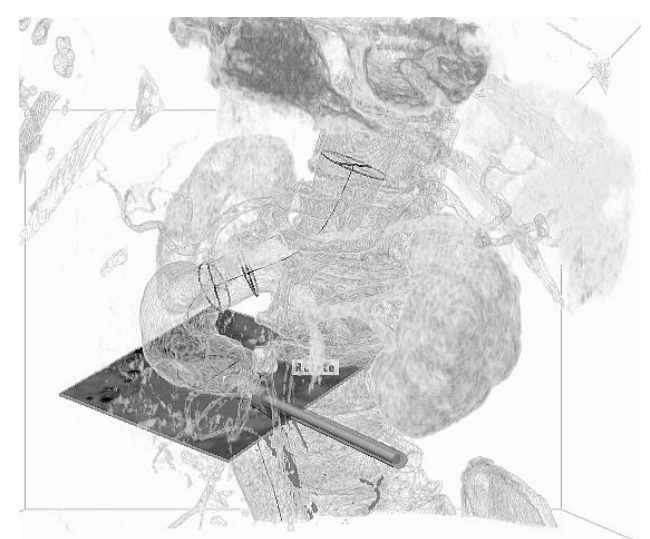

(a)

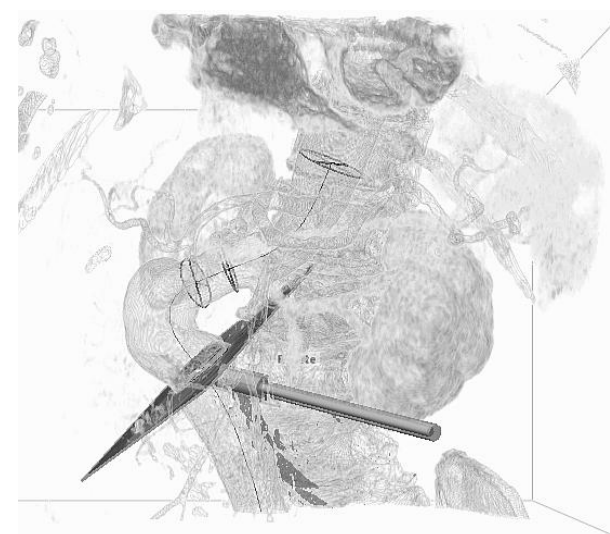

(b)

Figure 6: Rotating the diameter ellipse. (a) Before editing. (b) After editing. The orientation of the diameter ellipse is adjusted and the new measurements are recomputed. 


\subsubsection{Resizing and reshaping the diameter measurements}

Accuracy of the diameter measurements depends on the quality of the scan image. AngioDEX works well on varied data, including enhanced or poorly contrasted data, noisy data and even data with calcium deposits. In cases, where the fit is not optimal, the user can select the "Resize" tool and fine-tune the diameter measurements. Resizing changes only the size of the diameter measurement proportionately and the ratio of the maximum and minimum diameters does not change (Figure 7). By adjusting one axis of the ellipse, the user effectively changes the shape of the ellipse and it can allow the fitting of even odd-shaped aortas (Figure 8).

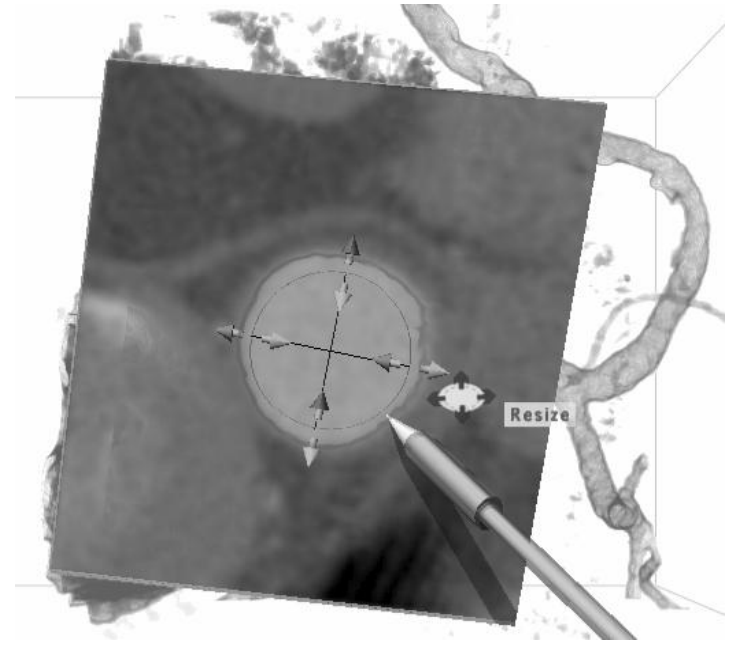

(a)

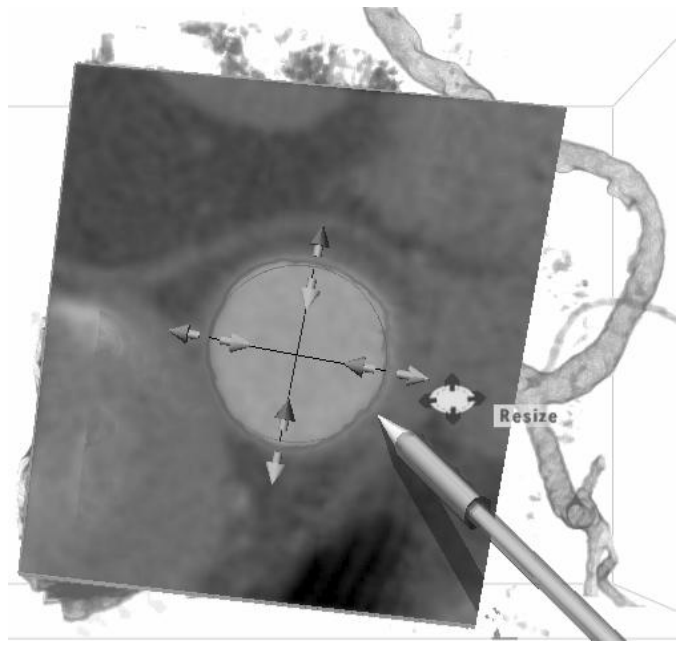

(b)

Figure 7: Resizing the diameter ellipse. (a) Before editing, the diameter ellipse is smaller than the blood vessel lumen. (b) After editing, the size of the diameter ellipse is enlarged.

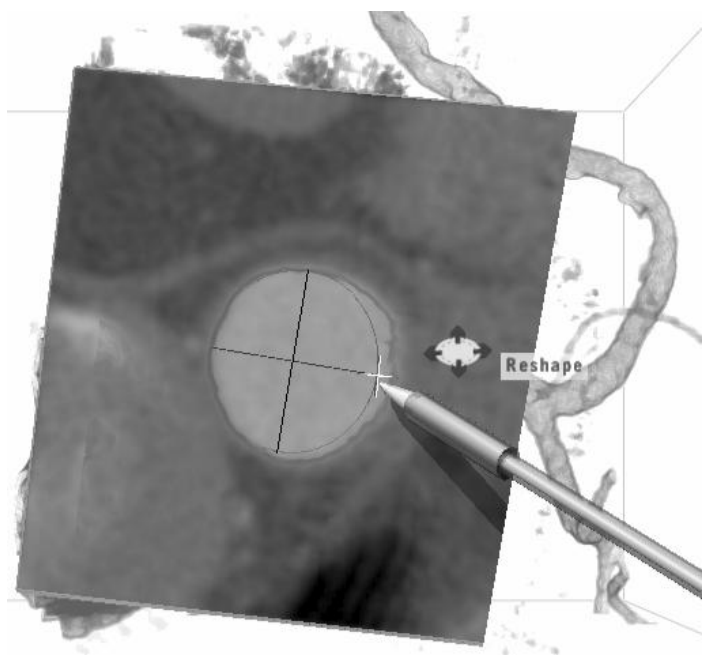

(a)

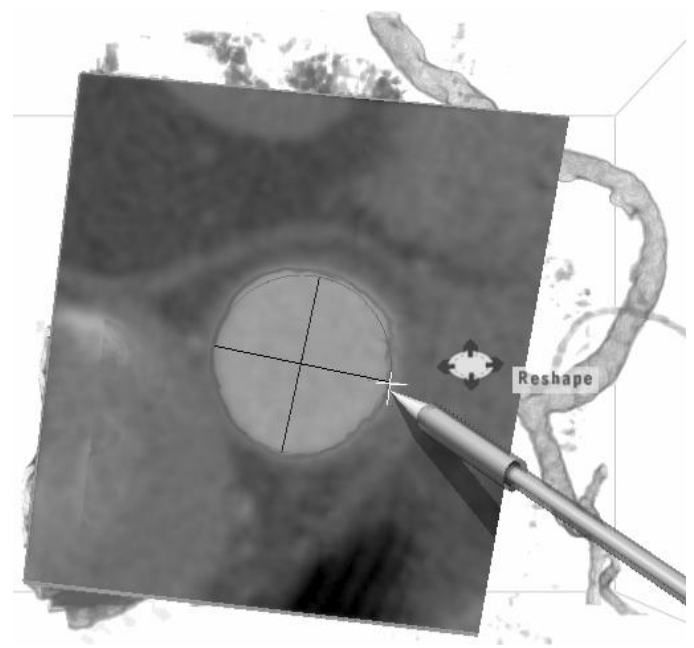

(b)

Figure 8: Reshaping the diameter ellipse. (a) Before editing, the diameter ellipse is smaller than the blood vessel lumen. (b) After editing, the short axis of the ellipse (blue line) is stretched. Hence the shape of the diameter ellipse is changed. 


\section{RESULTS}

We have tested the AngioDEX on CT and MR scan images. It works well on varied data, including both enhanced and some poorly contrasted data, and noisy data. The template can be correctly mapped onto very tortuous aorta. The method copes well with calcium deposits. We have also tested AngioDEX on some complicated AAAs. For example, some data have aneurysms on both the aorta and the iliac arteries. After initial placement of the points, it takes less than one minute to calculate all the measurements for a $512 \times 512 \times 342 \mathrm{CT}$ on a Windows $2 \mathrm{GHz}$ PC. Further manual editing of the centerline and diameter measurements add two to five minutes to the total time, depending on the time it takes the user. Table 1 shows the computation time of automatic measurement for some typical CT and MR scan data.

Table 1: Computation time of AngioDEX's automatic measurements

\begin{tabular}{|c|c|c|c|}
\hline Modality & Size (MB) & Voxel resolution & Time (seconds) \\
\hline MR & 4.07 & $208 \times 256 \times 80$ & 7.3 \\
\hline MR & 12.0 & $384 \times 512 \times 64$ & 20.9 \\
\hline $\mathrm{CT}$ & 50.75 & $512 \times 512 \times 203$ & 24.8 \\
\hline $\mathrm{CT}$ & 85.5 & $512 \times 512 \times 342$ & 47.5 \\
\hline $\mathrm{CT}$ & 216.0 & $512 \times 512 \times 864$ & 77.65 \\
\hline
\end{tabular}

AngioDEX is being clinically evaluated in several the radiology departments in the USA and Europe where over 100 cases have been planned so far.

\section{CONCLUSION}

Visualization of stent measurements and their relationships to the imaging data of the aorta is greatly improved by the use of a stereoscopic display. Stereoscopy allows to fuse the 3D measurements of the stent (length, diameters) with semi-transparent views of the wall of the lumen of the aorta. It also allows to look unambiguously to nonorthogonal cross-sections of the aorta along 'true' cross-sections of the vessel, in combination with volume rendered views. This visual complexity is easily comprehended thanks to the disambiguation that stereo offers. However, stereoscopy is not enough in itself. The display needs to be combined with a 3D interface. We have developed the Dextroscope for this purpose.

The stent planning application is especially useful when the aneurysm has extreme angulations or strong tortuosity. Even if the aneurysm is straightforward, it still offers advantages in further exploration of the spatial and structural position.

\section{REFERENCES}

1. Kelvin M.Baskin, Elvira V. Lang, Shereen Chang, etc., Three-dimensional Imaging of Abdominal Aortic Aneurysms Using Centerline-base Analysis: Techniques and Examples, Dept. of Radiology, University of Iowa, http://dpi.radiology.uiowa.edu/nlm/app/aorta/aaatech/AAAtech.html

2. J.D. Blankensteijn, Imaging Techniques for Endovascular Repair of Abdominal Aortic Aneurysms, Medica Mundi, 44:2, Nov 2002

3. Voxar 3D ${ }^{\mathrm{TM}}$ VesselMetrix ${ }^{\mathrm{TM}}$, http://www.barco.com/barcoview/downloads/VesselmetrixDown.pdf

4. A. Giachetti, M.Tuveri, G.Zanetti and E. Piccinini, Web Based 3D Quantitative Measurements of Abdominal Aortic Aneurysms, Center for Advanced Studies, Research, and Development in Sardinia, Uta, CARS 2001 
5. Kockro RA, Serra L, YeoTT, Sitoh YY, Chua GG, Ng H, Lee E, Lee YH, Nowinski W, Planning and Simulation of Neurosurgery in a Virtual Reality Environment, Neurosurgery, 2000, Vol. 46, No. 1, pp. 118-137

6. Serra L, Kockro RA, Chua GG, Ng H, Lee E, Lee YH, Chan C and Nowinski, WL, Multimodal Volume-based Tumor Neurosurgery Planning in the Virtual Workbench. MICCAI 98, Cambridge MA, USA, October 11-13, 1998, pp 1007-1016

7. Ralf Kockro, Could the Cath Lab One Day Use Virtual Reality to Prepare for Complex Procedures? Cath Lab Digest, vol.14, no.6, 2005 